\section{$\underset{\substack{\text { hommes } \\ \text { \& migrations }}}{ }$}

\section{Hommes \& migrations}

Revue française de référence sur les dynamiques

migratoires

$1324 \mid 2019$

Religion et discrimination

\title{
Fabrication des images et significations
}

\section{Anne Geslin - Beyaert}

\section{CpenEdition \\ Journals}

\section{Édition électronique}

URL : https://journals.openedition.org/hommesmigrations/8851

DOI : 10.4000/hommesmigrations.8851

ISSN : 2262-3353

\section{Éditeur}

Musée national de l'histoire de l'immigration

\section{Édition imprimée}

Date de publication : 1 janvier 2019

Pagination : 158-166

ISBN : 978-2-919040-44-5

ISSN : $1142-852 X$

\section{Référence électronique}

Anne Geslin - Beyaert, «Fabrication des images et significations », Hommes \& migrations [En ligne], 1324 | 2019, mis en ligne le 01 janvier 2019, consulté le 06 janvier 2022. URL : http:// journals.openedition.org/hommesmigrations/8851 ; DOI : https://doi.org/10.4000/ hommesmigrations.8851 


\section{RESEAU}

\section{Fabrication des images et significations}

\section{Anne Geslin-Beyaert,}

professeure de sémiotique à l'université Bordeaux-Montaigne,

directrice du laboratoire Mica (Médiations, informations, communication, arts).

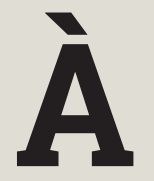

l'occasion de son Grand Festival, le Musée national de l'histoire de l'immigration a réuni ceux qui fabriquent et ceux qui lisent les images afin d'agir contre les discriminations qu'elles contiennent. L'invitation m'a amenée à de nombreuses réflexions. J'étudie les images et les affiches, pour ma recherche ou avec mes étudiants. J'ai donc eu l'occasion de mesurer la difficulté des exercices d'analyse et de fabrication, en travaillant particulièrement sur la figure du portrait ${ }^{1}$. Je pouvais penser avoir fait le tour de la question. Pourtant, la demande des associations qui participent au Grand Festival m'a révélé que le problème de la fabrication des images, en l'occurrence d'affiches, restait entier. J'ai tenté de reformuler les questions posées par la fabrication des images, en prenant appui sur des portraits et sur les affiches qui m'ont été adressées très gentiment par la graphiste Emmanuel Roule, animatrice des ateliers. Nous avons essayé de cerner la spécificité de la démarche des participants qui luttent contre les discriminations, et donc sa difficulté.

Je me suis appuyée sur le grand principe de la sémiotique: se demander, non pas ce que les images signifient, mais comment elles signifient, comment elles fonctionnent pour ainsi dire. Agir contre les discriminations au travers de l'image revient à recommander l'autre à l'attention, à s'attacher à des personnes victimes de stéréotypes pour essayer de modifier les relations que nous avons avec elles. Cette recommandation passe, la plupart du temps, par la représentation d'un autre dont on reproduit la relation, à travers le simulacre de l'affiche. L'image permet de faire comme si j'entrais en relation avec lui.

\section{Le portrait}

Le portrait s'offre comme un dispositif formidable car il extrait l'autre de son environnement pour le prendre à soi. Les portraits les plus efficaces présentent

1. Voir Anne Geslin-Beyaert, Sémiotique du portrait. De Dibutade au selfie, Paris, De Boeck supérieur, 2017. 
la figure sur un fond neutre ${ }^{2}$. Ils procèdent en fait à une extraction qui transforme l'environnement en un fond et le visage en une figure. Cette extraction optimise la relation entre la figure et le fond, en offrant la figure à l'attention de son interlocuteur. Les milliers ou millions de portraits que nous avons vus ont banalisé cette procédure, nous la donnant comme une évidence. Mais les évidences sont souvent des constructions. Il faut prendre cette double objectivation en considération (l'environnement devient un fond et le visage devient une figure). Les portraits regardent leur observateur et celui-ci les regarde (portrait collectif où chacun regarde l'observateur). C'est un geste d'humanité ${ }^{3}$ par lequel le producteur de l'image prend en considération l'autre et le pose comme un alter ego possible.

\section{Histoire des arts et portrait}

Tous les êtres humains n'ont pas reçu cet éloge du portrait. Les esclaves en sont, pour ainsi dire par définition, privés car, s'il est signe de considération, le portrait est aussi signe de liberté. Le Musée des beaux-arts de Bordeaux présente deux représentations atroces d'esclaves travaillant sur le port, traités comme un enchevêtrement, presque un engrenage de corps vus de très loin comme s'ils composaient d'immenses machines.

Le Musée d'Aquitaine à Bordeaux expose plusieurs portraits de « nègres de maison » parés de beaux costumes, posant à côté d'une dame. Il ne s'agit pas de portraits individualisés mais de représentations d'un prototype du Noir souriant gentiment, parés de beaux atours, toujours un peu plus petit que sa maîtresse. Un portrait véritable attire l'attention. Son intitulé « Portrait d'un jeune homme » suffit, par l'indication d'un changement de catégorie, à exemplifier la «promotion» de son modèle

16 Le portrait s'offre comme un dispositif formidable car il extrait l'autre de son environnement pour le prendre à soi. Les portraits les plus efficaces présentent la figure sur un fond neutre. dont le statut d'esclave se trouve ainsi dénié. Le portrait témoigne du changement de statut du sujet, de sa reconnaissance par une communauté ${ }^{4}$, à moins que, suivant le principe de l'efficacité symbolique, il n'accompagne cette transformation et «fasse être » dans ce nouveau statut.

La peinture occidentale n'a certes pas totalement ignoré les personnes noires. Avant d'être admises dans le tête-à-tête 《je»/《tu», elles étaient une figure parmi d'autres, présentée d'abord à la marge et de profil dans une composition, à moins que le peintre ne la valorise par des contrastes appuyés pour lui offrir le plus beau rôle. Ainsi, Breughel accorde au roi Gaspard un statut d'observateur

2. Jean-Luc Nancy, Le regard du portrait, Paris, Galilée, 2000.

3. Yves Citton, Gestes d'humanité. Anthropologie sauvage de nos expériences esthétiques, Paris, Armand Colin, 2012.

4. Sans doute s'agit-il d'un affranchi ou du fils d'un maître blanc. 


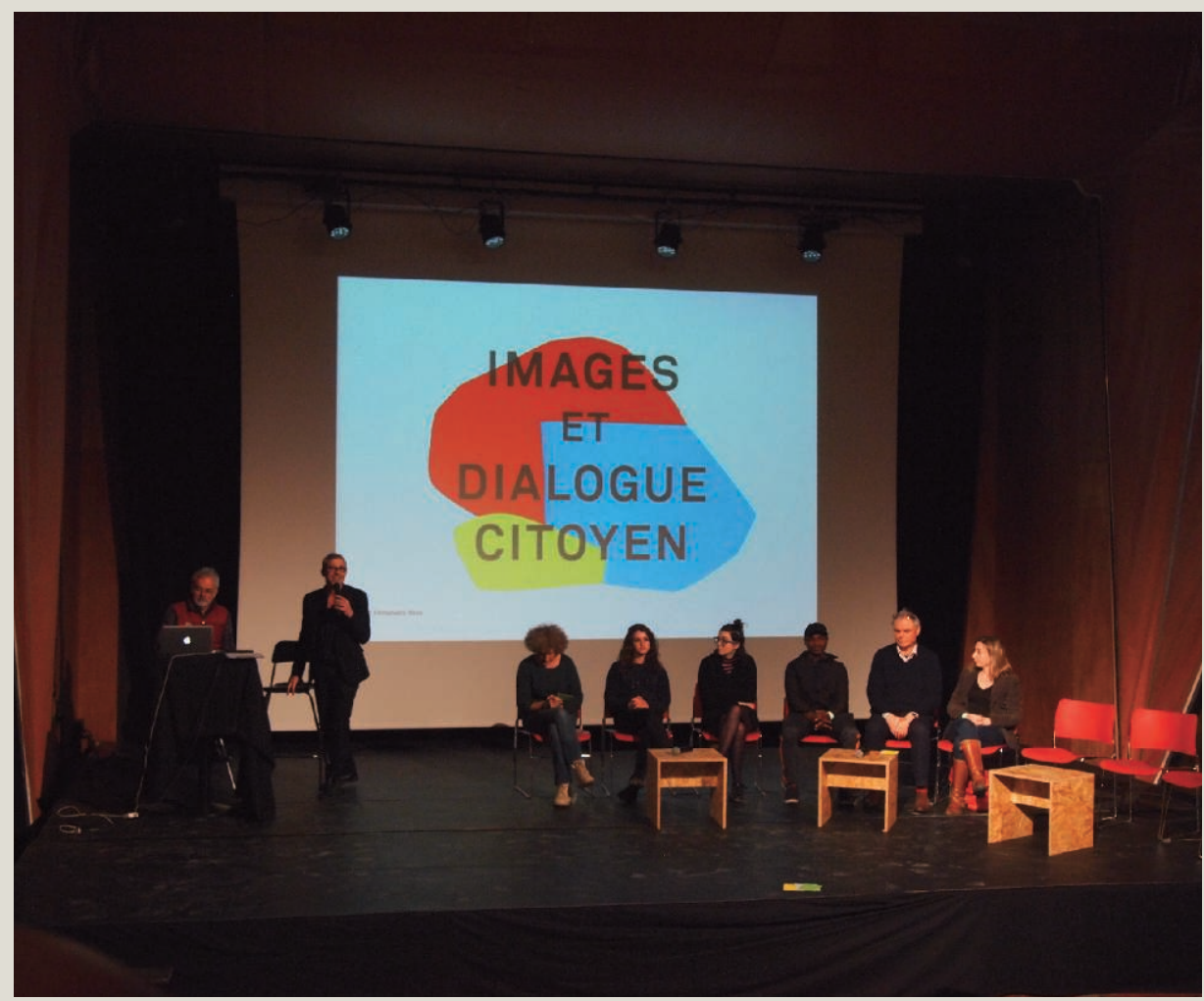

- Débat Images et dialogue citoyen, au MNHI, le 20 mars 2018 avec Agoralab, dans le cadre du Grand Festival. (c) Anne Volery.

délégué dans la scène de l'Adoration des mages ${ }^{5}$. Mais cette représentation parmi les figures n'est qu'un tout premier pas vers la reconnaissance. En 1800, Marie Guilhelmine Leroux-Delaville consacre un beau portrait à une jeune femme noire, souvent considéré comme le symbole de l'abolition de l'esclavage par la Révolution française. Retenant que cette abolition ne fut pas suivie d'effets - le décret déclarant l'abolition de l'esclavage de 1794 n'empêcha pas sa poursuite et son rétablissement par Napoléon pour les colonies françaises -, le portrait ne valide pas une considération sociale acquise, mais témoigne plutôt d'une invitation, d'une demande de reconnaissance adressée par le peintre à son époque. Il problématise seulement l'admissibilité. Le portrait peint par Marie Guilhelmine Leroux-Delaville trahit d'ailleurs l'ambiguïté de cette reconnaissance sociale. Il représente certes son modèle dans la pose d'une femme de la haute société, joliment vêtue, conformément aux stéréotypes du genre, mais avec un sein dénudé qui dévoile l'hypocrisie en renvoyant le modèle à un « naturel » exotique, à moins qu'il en fasse un symbole de liberté. 


\section{Le portrait photographique}

Le portrait peint recommande toujours à l'attention. Peut-on en dire autant du portrait photographique? Certains portraits, par exemple le portrait mondain, prolongent cette tradition et, historiquement, reprennent la scénographie du portrait peint ${ }^{6}$. Mais ce n'est pas le cas de la photographie ethnographique, ni de celui du portrait anthropométrique qui présente les personnes comme des objets mesurables en plaçant les têtes de face et de profil, les deux positions étant associées à différentes mesures du corps et aux empreintes digitales.

Avec ce genre photographique, nous apercevons déjà que l'image peut, en fonction de l'orientation de la tête et de corps, en fonction de certains éléments figuratifs, modifier, déterminer la relation au modèle. L'image en tout cas n'est pas neutre, le portrait n'est jamais neutre. Cela ajoute à la difficulté, utopie de la représentation de l'autre.

Ce qui est possible pour une photographie (Henno, Jacob Rijs), c'est de tourner le dos, c'est nier de la relation, tout comme de fermer les yeux. On ne peut renier la mise en relation, ou alors la problématiser? Ce n'est pas le cas du profil qui impose seulement un change-

Représenter de face ou de profil est donc un acte de langage qui valorise ou péjore l'autre et, dans un groupe, permet de distinguer les personnages principaux et secondaires en attribuant des statuts distincts.. ment de statut. Le profil supprime l'interaction mais transforme en un objet de contemplation un sujet tout de même susceptible d'interagir avec une autre personne située dans le hors-champ.

Avec le portrait anthropométrique, nous avons vu la juxtaposition d'un visage de face et de profil. Ceci nous renvoie à un système très souvent commenté ${ }^{7}$ (Meyer Schapiro). Une étude de portraits ou de représentations de groupe révèle que la personne de face correspond au pronom personnel «je» et la personne de profil à un «il » ou un « elle ». Ces remarques portent sur les représentations de groupe, celles de Jésus parmi les apôtres et plus précisément de représentations de la Cène. Cette orientation du corps accompagne d'autres dispositifs scénographiques: Jésus est de face et au centre. L'orientation de trois-quarts des visages des apôtres permet de faire circuler notre regard de proche en proche. Judas est de profil, en bout de table (avec des cheveux roux) et ramène notre regard vers le centre de la table.

Représenter de face ou de profil est donc un acte de langage qui valorise ou péjore l'autre et, dans un groupe, permet de distinguer les personnages principaux et secondaires en attribuant des statuts distincts. Il faut considérer l'opposition face/profil comme une dichotomie qui détermine un système de valeurs qui évolue avec le temps, au gré des genres et des usages sociaux.

6. Walter Benjamin, «Petite histoire de la photographie », in Euvres II, traduction française par Maurice de Gandillac, Rainer Rochlitz et Pierre Rusch, Paris, Gallimard, 2000.

7. Meyer Schapiro, «Face et profil comme formes symboliques», in Les mots et les images, trad. française, Paris, Macula, 2000. 


\section{La photo de presse}

La photo de presse représente fréquemment les chefs d'État de profil, en suivant le modèle de l'effigie romaine, et produit un effet de sens d'intronisation. À l'inverse, la photo anthropométrique a connoté la photo d'identité. L'absence de sourire et de signes particuliers fait une "tête d'assassin » ou de terroriste.

L'orientation des corps exprime la façon dont nous considérons l'autre, son statut dans un groupe, ce qu'il vaut dans ce groupe. La circulation du regard, guidée par l'orientation du visage de face (immobilisation), de profil (direction vers l'avant, comme une flèche) ou de trois-quarts (direction vers l'avant mais à proximité), permet aussi de répartir la compétence dans le groupe, la part que chacun y prend. Exemple, le portrait de groupe hollandais où tous les visages sont de trois-quarts, où chacun est individualisé par son vêtement sans qu'aucun ne reçoive la valorisation du centre ou d'un vêtement qui le mettrait en valeur. Le portrait de regroupe représente la forme de vie égalitaire de la société hollandaise et c'est le regard du spectateur qui actualise cette forme de vie et, en circulant, valorise chaque visage à son tour en lui donnant exactement la même importance.

Cette organisation égalitaire pourrait être comparée à celle de ce tableau de Giotto où l'on cherche en vain un visage de face, qui se trouve masqué par les deux visages de profil de Jésus et de Judas. Il faut remarquer par quels moyens figuratifs Giotto désigne Jésus à notre attention : par son auréole, une stature légèrement plus haute et un profil aquilin dont la beauté contraste avec la laideur du profil de Judas. Au-delà des ressemblances de leur représentation, fonction de la mimésis, les portraits peuvent imposer une grille de lecture axiologique, un système de valeur, dire comment il faut considérer l'autre. Cette grille de lecture est d'autant plus lisible qu'elle se fonde sur des contrastes, donc des portraits collectifs, des scènes narratives.

\section{Présence/distance du sujet}

Le portrait peut aussi définir différemment l'autre, en faisant de lui un corps éloigné ou un alter ego. Cette différence est donnée par la distance d'interaction. L'anthropologue Edward Hall a distingué quatre distances d'interaction qui organisent la vie sociale: la distance intime, personnelle, sociale et publique. Sans entrer dans le détail, on rapporterait la distance classique du portrait à la distance personnelle qui s'étend entre $45 \mathrm{~cm}$ et $125 \mathrm{~cm}$. C'est cette distance que reproduisent la plupart des portraits. Cette «bonne distance » permet de concilier les informations cognitives et affectives. En même temps, je peux observer tous les détails de l'apparence de l'autre et comprendre ses émotions. « L'âme du modèle commence à transparaître ${ }^{8}$ », dit Hall. Cette distance prédispose à l'empathie. 
S'écarter de cette distance personnelle, c'est prendre un double risque : vu de trop loin, l'autre n'est plus qu'une figurine. De trop près, lorsque je suis trop près de sa peau, celle-ci renvoie à une certaine étrangeté, susceptible de multiples interprétations. À la bonne distance, l'autre est restitué dans son volume exact. Il n'y a pas de basculement dans l'imaginaire et je peux le considérer comme un alter ego, semblable à moi.

Si on saisit bien les commodités de cette distance personnelle pour notre recommandation de l'autre, nous avons tous en tête des dérogations, des rapprochements ou des éloignements porteurs de sens.

À propos du cinéma, Gilles Deleuze décrit le «gros plan» comme une image-affection qui sensibilise tout le film ${ }^{9}$. Du rapprochement à l'éloignement extrême, différents scénarios sont possibles pour interroger la relation. Une magnifique scène des Misfits de John Huston réussit à rendre sensible l'interaction en procédant par un éloignement extrême de Marilyn Monroe dans le paysage désertique. Elle est hors d'elle et hurle dans le désert, et la disproportion de son petit corps dans le grand paysage produit un effet de fragilité particulièrement expressif.

\section{La fonction métonymique du portrait}

Il faut par ailleurs évoquer une caractéristique assez étrange du portrait, assez peu commentée, qui fait qu'un individu représente toujours, au-delà de lui-même, une catégorie, une classe. Cette capacité très intrigante apparaît avec acuité dans la série de portraits d'August Sander, mais aussi dans les portraits de Thomas Ruff qui ont rénové le portrait contemporain dans les années 1980. Sander représente un certain concierge, un certain artisan, un certain membre des Jeunesses hitlériennes mais, aussitôt photographié, celui-ci devient le parangon d'une catégorie. Non plus un concierge mais le concierge. Idem pour les étudiants photographiés par Ruff qui

(6) Le portrait peut aussi définir différemment l'autre, en faisant de lui un corps éloigné ou un alter ego. Cette différence est donnée par la distance d'interaction. se prêtent à la généralisation.

Tous les portraits produisent-ils cet effet de généralisation? Certains éléments accentuent cet effet de sens : la frontalité, l'inexpressivité, une clarté absolue... Le portrait d'Evergon représente la mère de l'artiste frontalement dans une pose inexpressive. Elle devient ainsi la représentante d'une catégorie qui la dépasse : la vieille dame, la personne âgée et non plus $\mathrm{M}^{\mathrm{me}} \mathrm{X}$. Le portrait d'Evergon reprend les codes observés chez Sanders et Ruff, mais avec une dérogation intéressante : l'utilisation de la lumière rasante qui, dévoilant la texture de la peau, participe à la caractérisation d'une personne âgée tout en l'individualisant. On découvre ainsi l'importance de la lumière pour l'effet d'individuation ou de généralisation: une clarté absolue participe à la généralisation/une clarté relative individualise en dessinant un visage. 
L'orientation, associée à cette organisation lumineuse, permet d'opposer la tête et le visage, la tête de tout le monde et le visage qui n'appartient qu'à soi. Alberto Giacometti a sans doute fait les plus beaux commentaires sur cette opposition. Dans L'Atelier d'Alberto Giacometti ${ }^{10}$, Jean Genet explique qu'à l'issue des longues séances de pose, Giacometti lui tend le portrait qu'il a fait de lui et dit: comme vous êtes beau, pas plus beau qu'un autre après tout. Genet explique - et ceci est repris dans un autre texte - qu'il a compris alors que chaque être vaut exactement un autre. Il insiste bien sur le "exactement». L'image, le portrait est un acte de langage qui dit comment nous considérons l'autre.

\section{Les affiches}

Certaines affiches de la Licra assument pleinement la fonction métonymique de l'image. Les bébés habillés en balayeur ou en femme de ménage représentent toute une catégorie. La Licra assume cette propriété pour la confronter à la propension au stéréotype langagier et à la fatalité sociale. Rien ne prédispose les bébés noirs à devenir des balayeurs, à part le regard que nous portons sur eux. Mais cette capacité du portrait à inscrire dans une catégorie doit retenir notre attention car elle rejoint le stéréotype. Mais, en même temps, toute communauté produit des représentations, des stéréotypes qui lui permettent de partager les expériences. Il faudrait pouvoir considérer l'autre comme un individu en le débarrassant de tous les préjugés, alors que le partage des expériences tend à projeter une grille de lecture sur le monde, pour en partager l'expérience. Comme le souligne un article rédigé à propos du Bal des Noirs du carnaval de Dunkerque, les bonnes intentions ne suffisent pas: une catégorisation, et donc une réduction de l'autre, est toujours à l'œuvre.

L'affiche a une fonction communicationnelle différente de celle du portrait peint ou photographique. Elle ne représente pas seulement une interaction, mais assume cette fonction de recommandation de l'autre en y associant un message textuel qui prend le relais de l'image. Observons, à titre de comparaison, le fonctionnement d'une affiche électorale puis celui du portrait officiel du président de la République. C'est un programme visuel en deux temps.

Il y a deux grands types d'affiche électorale : le portrait de trois-quarts face ou le portrait de face ${ }^{11}$. Dans le premier, il n'y a pas d'interaction. Le candidat contemple son beau projet. Dans le second, toute l'argumentation s'inscrit dans une interaction construite par la photo: le candidat est de face et donne l'impression à chacun d'avoir une relation individuelle. Le regard de face perce la limite temporelle et spatiale entre les deux espaces (énoncé/énonciation) si bien que le candidat et l'observateur sont réunis dans le même espace-temps. Le « je » du portrait de face construit un «nous ». L'interaction étant installée, le texte argumente la promesse. Il situe aussi l'identité de cette personne par rapport à une identité collective ou individuelle, celle du parti et la sienne. Le

10. Jean Genet, L'atelier d'Alberto Giacometti, Paris, L'arbalète/Gallimard, 1997.

11. Roland Barthes, «Photogénie électorale», in Mythologies, Paris, Points, 2014 [1957]. 
portrait officiel illustre une autre dimension communicationnelle. À celui qui, quelques jours ou quelques semaines auparavant, n'était qu'un candidat, il doit donner les moyens de gouverner. Il met donc de la puissance dans les signes en s'écartant de la promesse. Le portrait n'est pas légendé. On découvre ainsi l'efficacité symbolique du portrait qui «fait être » dans une autre identité, en assimilant la personne à la fonction, en l'institutionnalisant donc, en montrant sa légitimité et sa puissance par des signes.

Les affiches qui nous intéressent répondent à une toute autre mission. Il s'agit de recommander un autre pour faire bouger les stéréotypes. L'affiche tend à problématiser une interaction sociale asymétrique, infamante, en la déplaçant, en imageant la scène. Il ne s'agit pas de scénariser une promesse comme dans une affiche électorale. Le registre argumentatif du message est différent. La difficulté est qu'on se situe dans un registre prescriptif: il ne faut pas/il faut, vous ne devez pas/vous devez... Le risque de ce ton moralisateur est de susciter une position de défi, de défiance, voire une réaction manichéenne, comme le montre la

L'affiche tend à problématiser une interaction sociale asymétrique, infamante, en la déplaçant, en imageant la scène. contre-publicité à celle de la Licra.

L'écueil ressemble un peu à celui qu'affrontent les pubs anti-alcooliques ou anti-drogues, surtout celles qui s'adressent aux jeunes. Le public peut vouloir, par défi envers ce qui s'apparente à une prescription, exercer sa liberté. On pourrait se demander s'il ne faut pas se démarquer d'une dimension morale, qui renvoie à une position de censeur, de judicateur, pour placer la réflexion dans un registre éthique qui conduit à prendre position vis-à-vis de la morale : le je prends position vis-à-vis du nous.

Une possibilité consiste à renvoyer la question à l'observateur, en profitant de l'effet de notoriété d'une personnalité qui va permettre de renverser la péjoration de la maladie (cela peut être n'importe qui): me trouveriez-vous aussi drôle si j'étais séropositive?

\section{Registre de la conviction versus de la persuasion}

Deux registres sont à distinguer : celui de la conviction qui consiste à donner des arguments - texte de loi mais on tombe dans la prescription, arguments quantitatifs pour LGBT qui met en avant le taux de suicide chez les homosexuels... - ou celui de la persuasion qui ne donne pas d'argument mais construit une évidence figurative qui séduit. Exemple, une affiche qui recommandait les personnes séropositives par un portrait en très gros plan sur la lèvre duquel était inscrit un message tout petit qui obligeait à s'approcher. Le message disait: un baiser ne donne pas le sida. Cela confirme que l'image, n'importe quelle image, est active : elle met le corps en mouvement.

Pourtant, l'image n'était accompagnée d'aucun discours ni chargée d'aucune émotion. Il s'agissait simplement, par les exigences contradictoires d'une distance de lecture de l'image et du texte, d'inviter à s'approcher. 
Lors de sa campagne de 2006, la Licra a choisi la conviction en s'écartant d'un message général pour localiser les discriminations et les associer à des situations qui sont autant d'empêchements pratiques liés au racisme quotidien: trouver un travail, trouver un logement, entrer dans une boîte de nuit.

En 2018, la Licra fonde sa campagne sur des aphorismes de grands auteurs, une façon d'échapper à tous les risques de la caractérisation. On ne nous présente pas un alter ego. Des visages en arrière-plan, très pixellisés, ne facilitent pas l'interaction mais fonctionnent comme une référence à une personne, à la personne d'un auteur. Son texte est au premier plan et suggère une grande prise de hauteur par sa dimension poétique. Il nous éloigne de toutes ces situations pratiques de discrimination. Il s'agit d'une facilité. Cependant, cette distance généralisante et poétique peut faire adhérer au message, par sa dimension esthétique, la beauté de l'aphorisme, mais aussi par le soutien apporté par ce grand auteur: je ne suis pas seul à vouloir lutter contre les discriminations mais, en même temps, je vérifie que le problème est ancien, qu'il existe partout. Ainsi, je m'inscris dans une grande famille.

En étudiant de plus près ces affiches, différentes valeurs se distinguent: la solidarité (《 Je suis de la couleur de ceux qu'on persécute 》, Lamartine), mais aussi la combativité qui n'est pas directement associée à la solidarité vis-à-vis des personnes discriminées, n'est pas rapportée au combat antiraciste (« Celui qui combat peut perdre mais celui qui ne combat pas a déjà perdu », Bertold Brecht). C'est la signature de la Licra qui associe ce message à la lutte antiraciste. Ceci confirme que le message rencontre d'autres messages, une épaisseur de discours que nous avons en mémoire, qu'il va connoter à son tour.

Pour ne pas conclure, je souhaite mentionner néanmoins deux difficultés. Tout d'abord, un problème de catégorisation. Pour partager nos expériences, pour vivre ensemble, nous catégorisons le monde, nous le faisons entrer dans de grandes catégories. Or cette démarche devient problématique lorsqu'elle concerne des êtres humains: elle crée des stéréotypes. Il s'agit de savoir comment en sortir.

Le second problème rencontré est celui de la tonalité du message: il faut pouvoir poser un cadre légal, rappeler la loi qui protège les personnes discriminées. Mais le ton moralisateur peut être contre-productif. Pour s'en dégager, des formulations rhétoriques astucieuses sont nécessaires.

Une possibilité serait aussi de laisser l'observateur fabriquer l'image. L'image, toutes les images réclament notre participation, parce qu'elles sont des réductions, au moins de trois dimensions en deux dimensions, parce qu'elles imposent un point de vue, transforment des données qui ne sont pas seulement visibles en visible, et poussent l'observateur à déployer un visuel au-delà du visible.

Dans un tout autre registre, c'est ce qu'a bien compris la campagne de McDonald's qui supprime tout logo de ses affiches et propose à l'observateur d'investir l'image par ses expériences passées, son imaginaire de la marque. Cela suppose que l'observateur puisse solliciter un imaginaire déjà là. Il serait ainsi possible de prendre le parti de la suggestion, tout en convoquant l'arrièreplan juridique des discriminations. 